\title{
ELECTRON SPIN POLARIZATION IN FIELD EMISSION FROM NICKEL: EFFECTS OF SURFACE ADSORBATES औै
}

\author{
N. MÜLLER \\ Max-Planck-Institut für Plasmaphysik, D-8046, Garching bei München, Germany \\ and Sonderforschungsbereich 128
}

Received 6 August 1975

\begin{abstract}
Spin polarization of electrons field emitted from certain faces of more or less clean nickel is observed. The preferential spin direction is proved to depend on the crystal direction and on the adsorbate contamination of the face.
\end{abstract}

This letter reports measurements of the spin polarization $P$ of electrons field emitted from Ni tips; these raise doubts about the conclusion drawn from previous experiments with emission of spin polarized electrons from $\mathrm{Fe}, \mathrm{CO}$ and $\mathrm{Ni}$, and indicate magnetic interactions in adsorption of $\mathrm{H}$ on $\mathrm{Ni}$.

It has been claimed that in different experiments the spin density of the electronic states near the Fermi level at metal surfaces has been successfully measured:

(a) Photoemission [1] and (b) junction tunnelling [2] from polycrystalline films of $\mathrm{Fe}, \mathrm{CO}$ and Ni preferred majority spins $(P>0)$, which for $\mathrm{Ni}$ and $\mathrm{Co}$ were interpreted to be in contrast to the band model of ferromagnetism. (c) Field emission (FE) from $\mathrm{Ni}$ $[3,4]$ proved to be of little use because there was a peculiar rotation of the polarization vector. New measurements with $\mathrm{Fe}$ give stable emission with $P>0$ as well as with $P<0$ [5]. (d) Electron capture by deuterium ions $[6,7]$ gave a sign of $P$ which is consistent with band structure calculations, but $P$ is surprisingly high.

In all these experiments the emitting faces were not sufficiently well defined: under the given experimental conditions the electronic surface may be disturbed by adsorbates, especially $\mathrm{H}$, and with the exception of (d), the crystallographic faces from which the electrons come are unknown.

By comparison in our experiment, the state of the

47 Parts of this paper were presented at the Conference on Physical electronics, Penn. State University, June 1975, Bull. Am. Phys. Soc. II, 20 (1975) 859 T III, 4 (there is a printing error in the abstract: read 120-directions instead of 122). emitter is controlled by the FE pattern, and in performing the measurements of $P$, electrons emitted from certain faces of the tip are selected by positioning a certain part of the FE pattern onto a probe hole in the fluorescent screen. A schematic view of our apparatus is presented in ref. [8]. The magnet is now a static one. The vacuum conditions are improved, but the partial pressure of $\mathrm{H}_{2}$ is still of the order of a few $10^{-11}$ torr. During baking and after admission of gas the system is pumped by a turbomolecular pump and during FE work by an electrostatic ion pump (NRC Orb-Ion). To have the easy direction of magnetization, namely the 111 axis, near the tip axis, 110 and 111 oriented tips made of $4.5 \mathrm{~N}$ grade $\mathrm{Ni}$ are used [9]. The cleaning procedure applied to the tips is common in LEED and ion scattering: heating, Ne bombardment, and annealing [10]. With the 110 oriented tips, patterns indicating a nearly clean tip we:e obtained (fig. 1a,b,c); with 111 oriented tips the patterns suggested the presence of adsorbates (fig. 1d). Measurements were performed with different, more or less clean tips at room and liquid nitrogen temperature in magnetic fields of $0.6-2.5 \mathrm{kOe}$. We obtained the following results:

(1) $P=0$ is found in FE from Ni. The rotation of the polarization vector claimed in refs. $[3,4]$ is not verifiable by experimental evidence.

(2) The preferential spin direction depends on the crystallographic direction of the emitting face and the contamination of this face. A certain adsorbate, probably $\mathrm{H}$, reverts the sign of $P$ at faces between $\{110\}$ and $\{111\}$.

There was no dependence on the temperature and magnetic field in the observed region. Examples of (1) 


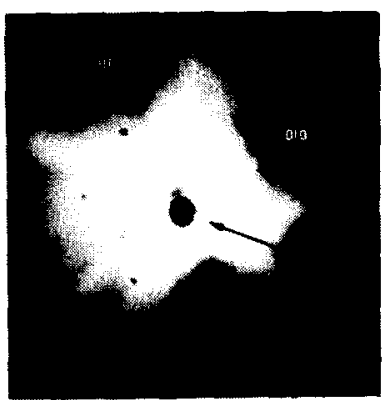

(a) $P=+2.2 \% \pm 0.8 \%$

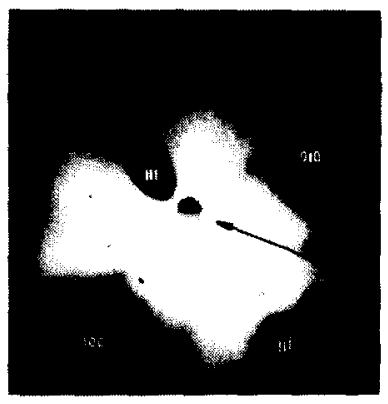

(b) $P=-4.5 \% \pm 1 \%$

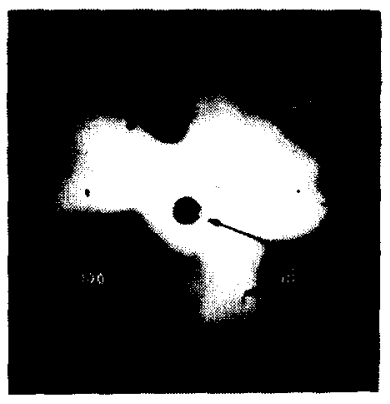

(c) $P=+3 \% \pm 1.2 \%$

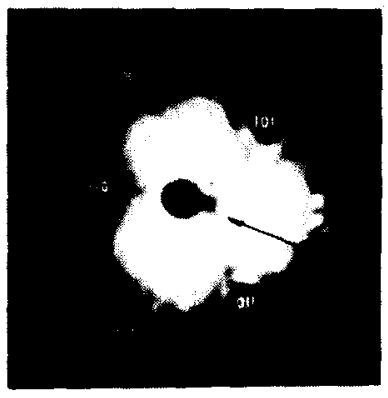

(d) $P=-7 \% \pm 1.5 \%$

lig. 1. (a)-(c) 110 oriented tip, magn. field $0.6 \mathrm{kOe}$, (a) high partial pressure of $\mathrm{H}_{2}$, (b) and (c) partial pressure of $\mathrm{H}_{2}$ improved. (d) 111 oriented tip covered with adsorbates, magn. field $2.5 \mathrm{kOe}$, total pressure better than $3 \times 10^{-11}$ torr. The arrow points at the probe hole.

are given in fig. 1a-d. Because it is hard to detect weak adsorption of $\mathrm{H}$ on $\mathrm{Ni}$ in $\mathrm{Fe}$ patterns [11], (2) is concluded from the following significant behaviour in time and temperature: A few hours after the end of bakeout the tip was subjected to a cleaning process. Before cleaning the total pressure of residual gas was about $5 \times 10^{-11}$ torr, this consisting mainly of $\mathrm{H}_{2}$. In the presence of the FE pattern in fig. la, i.e., with a nearly clean tip, $P>0$ was detected on all faces. After prolonged pumping before the cleaning process the total pressure was below $4 \times 10^{-11}$ torr and the partial pressure of $\mathrm{H}_{2}$ was lower. With a similar FE pattern first $P=-4.5 \% \pm 1 \%$ was detected between the 110 and 111 faces of the tip (fig. $1 \mathrm{~b}$ ); after about 10 min $P$ vanished, and after mild annealing $P=$ $-3.3 \% \pm 1.2 \%$ appeared. Between the 110 and 100 faces (near $\{120\}$ ) $P>0$ was always observed (fig. ic).

An improved examination of the $\mathrm{H}$ adsorption with a tip cleaned with the aid of field desorption is under preparation. But even the results obtained so far make it necessary to point out that results for the emission of spin polarized electrons from metal surfaces should be compared with theories only if the system has been sufficiently defined. This criterion is not adequately fulfilled in the experiments (a)-(d) mentioned above.

Thanks are due to Dipl.-Phys. K. Ertl for discussions and assistance, to Dr. W. Eckstein, Dr. H.
Vernickel, and Professor D. Menzel for discussions and critical reading of the manuscript.

\section{References}

[1] G. Busch, M. Campagna and H.C. Siegmann, Phys. Rev. B4 (1971) 746, see also review of all experiments in H.C. Siegmann, Phys. Repts. 17 (1975) 37.

[2] P.M. Tedrow and R. Meservey, Phys. Rev. B7 (1973) 318.

[3] W. Gleich, G. Regenfus and R. Sizmann, Phys. Rev. Lett. 27 (1973) 1066.

[4] G. Regenfus, Z. Phys. 269 (1974) 259.

[5] G. Chorbok, M. Hofmann and G. Regenfus, Verhandlg. DPG (VI) 10 (1975) 557, 0-7.

[6] M. Kaminsky, Phys. Rev. Lett. 23 (1969) 819.

[7] C. Rau and R. Sizmann, Proc. 5th Intern. Conf. on Atomic Coll. in Solids, Gatlinburgh, Tenn. (1973), eds. S. Datz, B.R. Appleton and C.D. Moak (Plenum Press, New York 1975) p. 295; see also Phys. Lett. 43A (1973) 317.

[8] N. Müller, W. Lckstein, W. Heiland and W. Zinn, Phys. Rev. Lett. 29 (1972) 1651.

[9] Tips are supplied by FEICO., P.O. Box 654, McMinnville, Oregon, USA.

[10] E. Taglauer and W. Heiland, Surf. Sci. 47 (1975) 234, and private communication.

[11] R. Wortmann, R. Gomer and R. Lundy, J. Chem. Phys. 27 (1957) 1099. 